\title{
Inhibition du développement de l'Aspergillus flavus par l'acide acétique: Analyse de trois expériences réalisées à Kinshasa- RD Congo
}

Umba di M'balu Joachim ${ }^{1}$, Masimango N. Thaddée. ${ }^{2}$ et Mvumbi Lelo ${ }^{3}$

${ }^{1}$ : Faculté de Médecine Vétérinaire, Université Pédagogique Nationale, B.P 8815, Kinshasa/Ngaliema

2: Faculté des Sciences Agronomiques, Université de Kinshasa, B.P. 190, Kinshasa XI

${ }^{3}$ : Faculté de Médecine, Université de Kinshasa, B.P. 190, Kinshasa XI

Corresponding author: E-mail:joachimumba@yahoo.fr, Cellphone:(243)82 22 48733)ou (243) 999992801

Mots-clés : Aflatoxines, Aspergillus flavus, acide acétique, milieu Czapek et mycélium

Keywords: Aflatoxins, Aspergillus flavus, acetic acid, Czapek medium and mycelium

Publication date 31/07/2020, http://m.elewa.org/Journals/about-japs/

\section{RÉSUMÉ}

L'Aspergillus flavus est un champignon cosmopolite, très répandu dans la nature et susceptible de contaminer plusieurs aliments. C'est un champignon qui fait beaucoup parler de lui depuis qu'on a découvert qu'il secrète de métabolites hautement toxiques, les aflatoxines, cancérigènes et exerçant d'autres effets nuisibles sur la santé des hommes et des animaux. En effet, les aflatoxines sont de métabolites toxiques secondaires biosynthétisés par certaines souches de micromycètes, notamment Aspergillus flavus. (En fait le terme est un moyen mnémotechnique pour dire : toxines d'Aspergillus flavus).Elles sont produites lorsque les champignons se trouvent dans des conditions de forte humidité relative (80-90\%) conjointement à une température élevée $\left(20-30^{\circ} \mathrm{C}\right)$.Les dégâts imputables aux aflatoxines sont nombreux aussi bien sur le plan de la santé (humaine et animale) que sur l'économie. En considérant que les mycotoxines ne peuvent jamais être complètement absentes ou éliminées des denrées alimentaires, divers moyens de lutte biologique, chimique ou physique empêchant le développement du champignon produisant l'Aspergillus flavus ont été essayés. L'objectif de ce travail est de faire connaître trois expériences de lutte des aflatoxines par l'acide acétique et de comparer si les résultats obtenus avec les extraits de caieux d'Allium sativum et d'écorces racinaires de Diospyros heterosictricha utilisé comme biopesticides pour inhiber la croissance mycélienne d'Aspergillus flavus. Il ressort des analyses que l'acide acétique exerce effectivement un pouvoir inhibiteur à des pourcentages différents sur le développement de l'Aspergillus flavus. La dose minimale efficace varie d'un auteur à un autre même lorsque les essais sont effectués dans de conditions comparables mais surtout en fonction de dilution. La dose minimale efficace d'inhibition d'Aspergillus flavus est située à $400 \mathrm{ppm}(0,04 \%)$ estiment certains auteurs. Par contre, d'autres pensent qu'elle est comprise entre $0,02 \mathrm{ml}$ à $15 \mathrm{ml}$.

\section{ABSTRACT}

Aspergillus flavus is a cosmopolitan fungus, widely distributed in nature and capable of contaminating several foods. It is a mushroom that has been talked about a lot since it was discovered that it secretes highly toxic metabolites, the aflatoxins, carcinogens and having other harmful effects on the health of humans and animals. Aflatoxins are secondary toxic 
metabolites biosynthesized by certain strains of micromycetes, notably Aspergillus flavus. (In fact the term is a mnemonic means to say: toxins of Aspergillus flavus). They are produced when the mushrooms are in conditions of high relative humidity $(80-90 \%)$ together with a high temperature $\left(20-30^{\circ} \mathrm{C}\right)$. The damage attributable to aflatoxins is numerous both in terms of health (human and animal) and in terms of the economy. Considering that mycotoxins can never be completely absent or eliminated from food, various means of biological, chemical or physical control preventing the development of the fungus producing Aspergillus flavus have been tried. The objective of this work is to make known three experiences of aflatoxin control by acetic acid and to compare if the results obtained with the extracts of cloves of Allium sativum and root barks of Diospyros heterosictricha used as biopesticides to inhibit the mycelial growth of Aspergillus flavus. Analyses show that acetic acid effectively exerts inhibitory power at different percentages on the development of Aspergillus flavus. The minimum effective dose varies from one author to another even when the tests are carried out under comparable conditions but especially according to dilution. The minimum effective inhibition dose of Aspergillus flavus is located at $400 \mathrm{ppm}(0.04 \%)$ believe some authors. On the other hand, others think that it is between $0.02 \mathrm{ml}$ to $15 \mathrm{ml}$.

\section{INTRODUCTION}

Les poussées scientifiques de ces dernières décennies ont souvent révélé que les dangers qui guettent la santé humaine (surtout dans les pays en développement) sont loin de disparaitre. En effet, de nombreuses maladies zoonotiques et de nouvelles formes d'infections ou d'intoxications voient régulièrement le jour, obligeant en même temps des précautions nouvelles. La lutte contre les mycotoxines dans les aliments est l'un des axes des travaux menés au niveau mondial dans le cadre du Codex alimentarius. Des règlements ou de recommandations ont édictés dans différents pays pour fiabiliser les échanges commerciaux des denrées alimentaires du point de vue de la sécurité sanitaire (Dragacci et al, 2005 et Nassima, 2010). Par ailleurs, des facteurs comme le climat, une mauvaise conservation et un mauvais stockage des aliments offrent les meilleurs conditions au développement et à la prolifération des certaines espèces microbiennes. De façon particulière, le cas de l'Aspergillus flavus est traité dans ce travail. En zones équatoriales et tropicales, l'humidité et la chaleur permettent une multiplication facile et rapide des moisissures. Ces champignons élaborent des métabolites dangereux appelés «aflatoxines» et qui manifestent une action cancérogénétique sur les organismes humains et animaux (Nikiema, 1993, Clavel, 2005). Ils sont classés pathologiquement comme hépatotoxines, néphrotoxines, vomitoxines et neuromusculotoxines dont certaines sont cancérigènes et mutagènes (FAO, 1989 Dans ces régions donc, le phénomène est si important qu'il exige toujours une attention particulière. La présence des aflatoxines dans les denrées alimentaires, spécialement dans les céréales et l'arachide, leurs conséquences sur la santé humaine, animale et même sur les économies appellent des études approfondies et une recherche des moyens de lutte efficaces. De nombreuses publications ont déjà accordé à la question des aflatoxines tout leur intérêt. Bon nombre d'études épidémiologiques, menées en Afrique notamment, ont démontré l'existence d'une corrélation positive entre l'ingestion d'aflatoxines par l'homme et le cancer du foie (Manima, 2005) et autres affections, telles que la microcéphalie, l'inophtalmie (Vesely et al, 1983, Sung - Hye Choet al, 2008). Dans le même temps, des pistes de solutions pour faire face aux conséquences néfastes qu'elles engendrent ont été envisagées: les moyens de lutte préventifs, c'est-à-dire ce qui empêchent le développement du champignon et la synthèse 
des aflatoxines ou encore les moyens de lutte curatifs qui s'orientent vers l'élimination de l'aflatoxine de la denrée où elle se trouve (Masimango, 1987). L'objectif de cet article est de passer en revue quelques travaux récents effectués sur l'action de l'acide acétique en rapport avec la croissance et le développement d'Aspergillus flavus. Les espèces d'Aspergillus sont très courantes dans l'environnement, principalement dans les sols et la végétation en décomposition, mais un certain nombre d'espèces sont également étroitement associées à l'alimentation humaine, en particulier les céréales et les noix (Pitt et Hocking, 1985a). Mais l'Aspergillus flavus est le premier champignon produisant une toxine incriminée et ayant donné le nom à un genre de champignons, Aspergillus, qui se développe sur divers milieux à la faveur de la chaleur et de l'humidité (Nikiema, 1993; Boucher et Nouaille, 2002). Le genre Aspergillus comprend, selon Golama (2005), près de 180 espèces réparties en 18 groupes suivant la structure de l'appareil reproducteur. Aflatoxines, sont aussi produites par d'autres espèces d'Aspergillus comme A. parasiticus (Masimango, 1987; DeWaele et Swanevelder, 2001; Mazoyer,
2002 ; Senani, 2010) et $A$. nominus. Par définition, les aflatoxines sont des mycotoxines ou des métabolites toxiques secondaires biosynthétisés par certaines souches de micromycètes (A.flavus et d'autres champignons apparentés), dans des conditions appropriées de température et d'humidité (Raemaekers, 2001 et Mazoyer, 2002). En outre, ces métabolites manifestent une action cancérogénétique fort élevée sur les animaux et sont élaborés par les moisissures à la faveur d'une forte humidité relative (80-90\%) conjointement à une température élevée $\left(30-35^{\circ} \mathrm{C}\right)$ (Umba, 2004). Les mycotoxines ont pour cause la dégénérescence des organes vitaux notamment chez les omnivores tels le porc et l'homme (Vesely et al, 1983 ; Sung-Hye Choet al, 2008). De par sa situation géographique, ses climats variés, la richesse de son agriculture, la RD Congo offre un champ de prédilection à la prolifération de moisissures et à la production d'aflatoxines. On peut s'attendre à ce que celles-ci contaminent les denrées alimentaires de ce pays tels que le riz, le maïs, les patates douces, les bananes, l'arachide, le sorgho et l'éleusine (Tableau 1) (Masimango, 1987 ; Umba, 2004 et FAO, 1989).

Tableau 1: Aflatoxines dans les aliments vendus sur les marchés de Kinshasa (RD Congo)

\begin{tabular}{|l|l|l|l|l|}
\hline Auteurs & Aliments & $\begin{array}{l}\text { Nombre d'échantillons } \\
\text { examinés }\end{array}$ & $\begin{array}{l}\text { Nombre d'échantillons } \\
\text { contaminés }\end{array}$ & $\begin{array}{l}\text { Aflatoxine } \\
\text { ppm }\end{array}$ \\
\hline Tshibangu (1972) & Arachides & 5 & $5(100 \%)$ & $3,3-88\left(\mathrm{~m}^{\prime} 20\right)$ \\
& Manioc & 6 & $2(33 \%)$ & $0,3-3\left(\mathrm{~m}^{\prime} 1,8\right)$ \\
\hline Kabeya (1973) & Arachides & 5 & $3(60 \%)$ & $0,1-2\left(\mathrm{~m}^{\prime} 0,73\right)$ \\
& Maïs & 29 & $3(10 \%)$ & $0,2-6\left(\mathrm{~m}^{\prime} 2,2\right)$ \\
\hline Mabiola (1973) & Manioc & 30 & $5(17 \%)$ & $0,05-0,2\left(\mathrm{~m}^{\prime} 0,17\right)$ \\
& Riz & 8 & $1(12,5 \%)$ & $0,016\left(\mathrm{~m}^{\prime} 0,016\right)$ \\
\hline
\end{tabular}

Source : Masimango, 1987.

Selon Masimango (1979), les aflatoxines sont présentes dans toutes les denrées consommées par la population congolaise mais la fréquence de contamination des aliments par les aflatoxines est plus grande dans le cas des arachides et du manioc et faible dans les autres cas notamment dans le maïs et le riz. En ce qui concerne la teneur en aflatoxine, les valeurs trop élevées : $88 \mathrm{ppm}$ sont atteintes pour les arachides et $17 \mathrm{ppm}$ pour les patates douces humides. Ce qui lui fait dire que de tels aliments doivent être écarté de la consommation. Les aliments en provenance des régions sèche et froide (le grand Kivu et le grand Katanga) sont moins fréquemment contaminés par les aflatoxines que ceux en provenance de parties du pays où règnent un climat chaud et humide (la ville-province de Kinshasa et l'ex-province 
Orientale). Le taux d'aflatoxines est généralement moins élevé dans les aliments en comparé à celui des régions chaudes et humides provenance des régions sèches et froides

(Tableau 2).

Tableau 2: Aflatoxines dans les aliments en provenance de diverses provinces de la RD Congo

\begin{tabular}{|l|l|l|l|l|}
\hline $\mathbf{N}^{\circ}$ & Aliments & Origine & Aflatoxines détectées & Aflatoxines $\mathbf{B}_{\mathbf{1}}$ ppm \\
\hline 1 & Patates douces humides & Kinshasa & $\mathrm{B}_{1}, \mathrm{~B}_{2}, \mathrm{C}_{1}, \mathrm{C}_{2}$ & 17 \\
\hline 2 & Arachides & Kinshasa & $\mathrm{B}_{1}, \mathrm{C}_{1}$ & 8 \\
\hline 3 & Cossettes de manioc & Kinshasa & $\mathrm{B}_{1}, \mathrm{~B}_{2}, \mathrm{C}_{1}, \mathrm{C}_{2}$ & 6 \\
\hline 4 & Cossettes de manioc & Province Orientale & $\mathrm{B}_{1}, \mathrm{~B}_{2}, \mathrm{C}_{1}, \mathrm{C}_{2}$ & 4 \\
\hline 5 & Arachides & Kinshasa & $\mathrm{B}_{1}, \mathrm{C}_{1}$ & 2 \\
\hline 6 & Farine de manioc & Kinshasa & $\mathrm{B}_{1}$ & 1 \\
\hline 7 & Farine de maïs & Kinshasa & $\mathrm{B}_{1}$ & 0,6 \\
\hline 8 & Farine de manioc & Kinshasa & $\mathrm{B}_{1}, \mathrm{~B}_{2}, \mathrm{C}_{1}, \mathrm{C}_{2}$ & 0,5 \\
\hline 9 & Bananes & Kivu & $\mathrm{B}_{1}, \mathrm{C}_{1}$ & 0,2 \\
\hline 10 & Farine de manioc & Province Orientale & $\mathrm{B}_{1}, \mathrm{~B}_{2}$ & 0,08 \\
\hline 11 & Farine de maïs & Province Orientale & $\mathrm{B}_{1}, \mathrm{C}_{1}$ & 0,03 \\
\hline 12 & Sorgho germé & Kivu & $\mathrm{B}_{1}, \mathrm{~B}_{2}$ & Traces \\
\hline 13 & Éleusine & Kivu & $\mathrm{B}_{1}, \mathrm{~B}_{2}$ & Traces \\
\hline 14 & Farine de manioc & Kivu & $\mathrm{B}_{1}$ & Traces \\
\hline 15 & Farine de manioc & Kinshasa & $\mathrm{B}_{1}$ & Traces \\
\hline 16 & Sorgho germé & Kivu & $\mathrm{B}_{1}$ & Traces \\
\hline 17 & Sorgho germé & Kivu & $\mathrm{B}_{1}, \mathrm{C}_{1}$ & Traces \\
\hline 18 & Sorgho germé & Kivu & $\mathrm{B}_{1}, \mathrm{C}_{1}$ & Traces \\
\hline 19 & Pomme de terre & Kinshasa & $\mathrm{B}_{1}, \mathrm{~B}_{2}, \mathrm{C}_{1}, \mathrm{C}_{2}$ & Traces \\
\hline
\end{tabular}

Source : Masimango, 1979.

Ces résultats montrent que les aflatoxines sont présentes en quantité variable, atteignant parfois des taux alarmants dans les denrées consommées par la population congolaise. Or, il est connu (Masimango, 1979) que les aflatoxines jouent un rôle dans la pathogénie du cancer primitif du foie, par ailleurs très fréquent en RD Congo, surtout chez les personnes de sexe mâle âgées de 30 à 40 ans. Ces données bien que fragmentaires faisant état des empoisonnements par des champignons, il exige d'approfondir les recherches visant comme ultime but, la suppression des aflatoxines dans les aliments (prévention, détoxication) ou leur inactivation vis-à-vis des systèmes vivants. Historiquement, il faut situer l'ingestion de macromycètes et micromycètes vers les années 1960. En effet, c'est à cette période qu'un élevage de dindons a été décimé dans le sud de l'Angleterre par un mal encore inconnu, il reste difficile d'en déterminer avec exactitude le foyer de naissance. Il a d'abord été imputé à des tourteaux d'arachides d'origine brésilienne et portait le nom de «Turkeys X disease ». Cependant, on s'aperçut rapidement au même moment que des hépatomes étaient présents dans un élevage des truites dans l'Idaho où l'aliment préparé à base de tourteaux de coton, de farine de poisson, de déchets de viande et de poudre de lait était aussi contaminé. De plus, à travers le monde, dans différents continents et pays, les basses-cours en proie à cette maladie mal connue étaient innombrables. Certaines données de la littérature rapportent que les aflatoxines sont, chimiquement, des composés organiques hétérocycliques. Elles ont une molécule caractérisée par la présence d'un noyau coumarique (d'où l'appellation flavacoumarines) (Masimango, 1978) et d'un noyau furfurolique. Suivant leur structure, on distingue deux groupes : les aflatoxines du groupe B ayant une 
fonction lactone et celles du groupe $G$ ayant deux fonctions lactones. Les aflatoxines du premier groupe ont un noyau pentacyclique carboné, celles du deuxième groupe un noyau hexacycliquehétéro-atome avec, en plus, un atome d'oxygène (Masimango, 1978). Ce qu'il convient cependant de retenir, c'est que le groupe de mycotoxines ainsi appelées constitue un ensemble d'une vingtaine des molécules dont quatre passent pour les formes les plus couramment rencontrées: $B_{1}, B_{2}, G_{1}$ et $G_{2}$ (Masimango, 1978 ; Bastiaanse $\mathrm{H}$ et Van Hove, 2006 et Senani, 2010). Elles sont distinctes par certaines caractéristiques comme on peut le constater dans le tableau 3.

Tableau 3: Quelques caractéristiques des aflatoxines majeures

\begin{tabular}{|c|c|c|c|c|}
\hline$\underbrace{\text { Aflatoxines }}_{\text {Caractéristiques }}$ & $\mathbf{B}_{1}$ & $\mathbf{B}_{2}$ & $\mathbf{G}_{1}$ & $\mathbf{G}_{2}$ \\
\hline Formule brute & $\mathrm{C}_{17} \mathrm{H}_{12} \mathrm{O}_{6}$ & $\mathrm{C}_{17} \mathrm{H}_{14} \mathrm{O}_{6}$ & $\mathrm{C}_{17} \mathrm{H}_{12} \mathrm{O}_{7}$ & $\mathrm{C}_{17} \mathrm{H}_{14} \mathrm{O}_{7}$ \\
\hline Poids moléculaire & 312 & 314 & 328 & 330 \\
\hline $\mathrm{T}^{\circ}$ de fusion ${ }^{\circ} \mathrm{C}$ & $268-269$ & $286-289$ & $244-250$ & $230-240$ \\
\hline Fluorescence en U V & Bleu pourpre & Bleu violet & $\begin{array}{l}\text { Verdâtre } \quad \text { ou } \\
\text { jaunâtre }\end{array}$ & $\begin{array}{ll}\text { Verdâtre } & \text { ou } \\
\text { jaunâtre } & \\
\end{array}$ \\
\hline D. L. 50 pour caneton d'un jour & 18 & 85 & 39 & 172 \\
\hline
\end{tabular}

Source : Masimango(1978); Bastiaanse H et Van Hove (2006) et Senani (2010)

Légende : -UV : rayons ultraviolets

-D.L. 50 : dose léthale 50, qui est « la quantité de substance toxique entraînant la mort de $50 \%$ des individus d'un lot, elle est généralement exprimée en mg de substance administrée par kg de poids vif de l'animal».

\subsection{Structure chimique des 4 aflatoxines majeures}

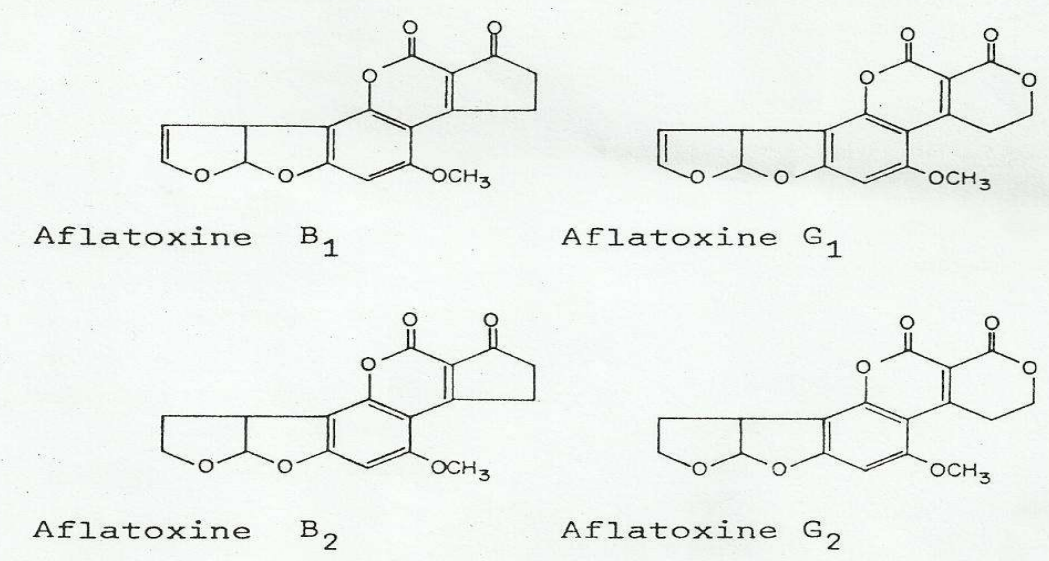

Source : Senani (2010)

L'indice 1 indique que le premier noyau garde son caractère furfurolique alors que l'indice 2 signale l'hydrogénation de la double liaison. Il est aussi utile de savoir que l'aflatoxine $\mathrm{B}_{1}$ est la plus virulente de toutes, quantitativement la plus importante et celle sur laquelle se sont concentrées la plupart des recherches sur les aflatoxines. Un dérivé phénolique de l'aflatoxine $\mathrm{B}_{1}$ apparu principalement sous forme conjuguée a été identifié dans l'urine du singe rhésus. Le groupement méthoxy de l'aflatoxines $\mathrm{B}_{1} \quad \mathrm{y}$ est remplacé par un 
groupement hydroxyle (Dalezios et al, 1971). Ce dérivé a été baptisé « aflatoxine $\mathrm{P}_{1}$ ».

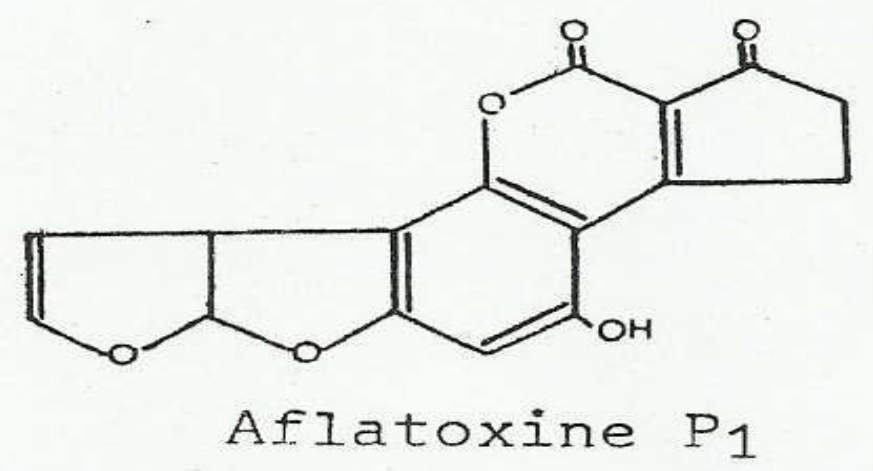

Source : Senani (2010)

Les meilleures conditions de développement pour les Aspergillus sont généralement offertes à la faveur conjointe d'une humidité relative de $80-90 \%$ et d'une température de $20-35^{\circ} \mathrm{C}$ (Masimango, 1978). L'on suppose que les conditions qui favorisent le développement d'Aspergillus sont celles qui permettent également une production appréciable d'aflatoxines. Ce qui fait que les régions tropicales et équatoriales constituent les zones de leur présence et leur distribution dans diverses denrées alimentaires de base. De ce qui précède, il en découle que les conditions et les milieux de production des aflatoxines sont aussi nombreux que diversifiés. De fait, les risques de contamination restent énormes et, partant, les conséquences virtuellement dévastatrices. Il convient de s'en rendre toujours compte afin de les prévenir à défaut de les écarter.

\section{MOYENS DE LUTTE CONTRE LES AFLATOXINES}

L'importance des études relatives aux moyens de lutte contre les aflatoxines dans les aliments, disent Masimango (1979) et Diem et al. (2016), n'est plus à démontrer au regard des pertes économiques que ces toxines occasionnent aux productions agricoles et aux élevages, de même que les dangers auxquels sont exposés les consommateurs des denrées contaminées. De nombreux travaux témoignent de l'intérêt accordé à ces moyens qui, du reste, sont soit préventifs, soit chimiques, soit physiques, soit biologiques (Masimango, 1978; Boucher et Nouaille, 2002, Luyila, 2006 ; FAO, 1989. Parmi les plus récents, l'on pouvait noter ceux qui se sont intéressés aux acides minéraux, notamment l'acide propionique et l'acide acétique (Muruhuka, 2004; Luyila, 2006; Khasa, 2006 ; Ivami, 2007); d'autres ont cherché à exploiter le potentiel de certains végétaux (Makuba, 2007; Diafuka, 2007; Pululu, 2007); d'autres encore ont essayé un antagonisme éventuel entre Aspergillus flavus et d'autres micro-organismes, en l'occurrence Propioni bactériumacne (Manima, 2007). D'une manière générale, la lutte peut s'organiser sur deux plans :

- Le plan préventif

- Le plan curatif

3.1 Moyens de lutte préventifs : La prévention peut être naturelle ou artificielle La prévention naturelle fait appel à la pratique de quelques règles élémentaires visant à empêcher la germination des spores de champignons. Ces règles consistent en de bonnes pratiques culturales, l'usage de semences saines, sans brisures, la récolte à maturité et à l'aide d'un matériel qui n'endommage pas la moisson, le séchage et le 
stockage dans les conditions excluant la possibilité de développement du champignon. La prévention artificielle a comme objectif, limité, à défaut d'inhiber, le développement du champignon et la synthèse de la toxine. Cette prévention recourt à des moyens physique tels que :

- le rayonnement gamma qui, appliqué aux souches toxinogènes, génère des mutants ayant perdu toute nocivité,

- des atmosphères impropres à la prolifération de micro-organismes : certains gaz ainsi que diverses combinaisons de dioxyde de carbone, de monoxyde de carbone, de diazote et de dioxygène sont défavorables à la germination du micro-organisme. Cette prévention artificielle recourt également à des moyens chimiques tels que l'usage des antifongiques ou de diverses autres substances chimiques pouvant contribuer avec des succès plus ou moins marqués à la lutte contre la présence du champignon et contre la biosynthèse d'aflatoxines (Masimango, 1987 ; Moll, M \& Moll, N, 2002). S'agissant de l'effet des acides organiques sur la croissance d'Aspergillus flavus et la production d'aflatoxines, différentes études, n’ont pas clairement établi, la relation entre les conditions du milieu et la concentration d'acide. Ainsi, cet article essaie d'apporter les informations sur l'effet inhibiteur de différentes concentrations d'acide acétique sur la croissance d'Aspergillus flavus (et la production des aflatoxines) pour ainsi aider à répondre aux questions suivantes :

- $\quad$ quelle est la dose minimale efficace qu'il faut conseiller?

- dans quelles conditions?

En 2006, Khasa, essayant l'acide propionique dans ses recherches, avait obtenu une dose minimale efficace égale à 333 ppm. L'acide acétique, acide de la même série que l'acide propionique est plus fort que ce dernier. Il s'avère que la dose minimale efficace pour l'acide acétique serait sans doute inférieure à 333 ppm, valeur obtenue par Khasa en 2006 pour l'acide propionique.

3.2 Moyens de lutte curatifs : Il s'agit de moyens mis en œuvre pour éliminer l'aflatoxine in situ :

- $\quad$ tri, décorticage sélectif

- détoxication: par usage d'acides, des bases, des sels.

\section{ESSAI D'INHIBITION DU DÉVELOPPEMENT DE L'ASPERGILLUS FLAVUS PAR L'ACIDE ACÉTIQUE}

L'acide acétique $(\mathrm{CH} 3 \mathrm{COOH})$ est un acide organique (acide éthanopïque) naturellement présent dans le vinaigre où il est responsable du goût amer. C'est un liquide incolore et inflammable. Son point de fusion se situe à $16,6^{\circ} \mathrm{C}$ et son point d'ébullition à $117,9^{\circ} \mathrm{C}$. Sa densité est de 1,049. L'acide acétique est aussi connu sous le nom d'acide acétique glacial car il devient solide à une température légèrement

\section{MATÉRIEL ET MÉTHODES}

\subsection{Matériel biologique : La souche} d'Aspergillus flavus engagée dans les travaux avait été isolée de substrats moisis et identifiée par les critères évoqués en 1.1. plus basse que la température ambiante. Dans le corps humain l'acide acétique est produit en outre par la consommation d'alcool : l'éthanol est converti en acétaldéhyde qui est alors converti en acide acétique est corrosive et doit être manipuler avec soin. Quoi qu'elle peut causer des brûlures ainsi que des dommages permanents à la bouche, au nez, à la gorge et au poumon.

5.2 Milieu de culture : C'est le Czapek qui a été utilisé comme milieu de culture. Sa composition est donnée au point 5.4

5.3 Acide acétique: L'acide acétique commercial ( $d=1,05 ; 99,5 \%)$ est la base de 
l'expérience d'essai d'inhibition du développement de l'Aspergillus flavus

\subsection{Préparation du milieu de Czapek}

Tableau 4 : Composition du milieu de Czapek

\begin{tabular}{|c|c|}
\hline Ingrédients & g/1 d'eau \\
\hline $\mathrm{NaNO}_{3}$ & 3 \\
\hline $\mathrm{K}_{2} \mathrm{HPO}_{4}$ & 1 \\
\hline $\mathrm{MgSO}_{4} \cdot 7 \mathrm{H}_{2} \mathrm{O}$ & 0,05 \\
\hline $\mathrm{KCl}$ & 0,5 \\
\hline $\mathrm{FeSO}_{4.7 \mathrm{H}_{2} \mathrm{O}}$ & 0,001 \\
\hline Saccharose & 100 \\
\hline Extrait de levure & 2 \\
\hline $\mathrm{H}_{2} \mathrm{O}$ distillée & 1000 \\
\hline
\end{tabular}

Les différents ingrédients de ce milieu ont été pesés puis dissouts dans 1 litre d'eau distillée. Après avoir soigneusement mélangé la solution, a été chauffée en agitant jusqu'à ébullition. Le milieu chaud ainsi préparé a été réparti en 2 lots de 5 flacons contenant chacun $200 \mathrm{ml}$. Les flacons ont été bouchés à l'ouate et recouverts de papier aluminium, et placés dans l'autoclave pour une stérilisation à $120^{\circ} \mathrm{C}$ pendant $20 \mathrm{~min}$. Après avoir été retirés de l'autoclave, ils ont été refroidis jusqu'à plus ou moins $40-50^{\circ} \mathrm{C}$.

- Préparation de l'inoculum (culture-mère) matériel d'expérience: La souche utilisée dans cette expérience appartient à l'espèce Aspergillus flavus (lot $\mathrm{n}^{\circ}$ ATCC 10124) ; elle est productrice d'aflatoxine $B_{1}$. Un prélèvement du milieu été ensemencé dans 200 $\mathrm{ml}$ du milieu Czapek et incubé à $37^{\circ} \mathrm{C}$. Après 7 jours d'incubation on obtient la culture mère (ou inoculum.

- Incorporation de l'acide au milieu de culture et ensemencement: A partir de la culture mère et sous une hotte à flux laminaire (Telstar AV-100) ont été ensemencés dans 5 flacons contenant chacun $200 \mathrm{ml}$ de Czapek. Dans un premier essai 0,$05 ; 0,10$; 0,$20 ; 0,25 \mathrm{ml}$ d'acide acétique $(\mathrm{d}=1,05 ; 99,5$ $\%$ ont été respectivement ajoutés à quatre flacons étiquetés, avec dates de

6 RÉSULTATS ET DISCUSSION Cette section présente les résultats relatifs à l'effet de l'acide acétique sur la croissance de l'ensemencement et d'addition de l'acide ainsi qu'une lettre correspondant au volume d'acide ajouté $(a=0,05 \mathrm{ml} ; \mathrm{b}=0,10 \mathrm{ml} ; \mathrm{c}=0,20 \mathrm{ml}$ et $\mathrm{d}=0,25 \mathrm{ml})$. Dans le second essai, d'autres volumes du même acide ont été respectivement ajoutés à quatre autres flacons aussi étiquetés, comme dans le premier cas mais avec $a=0,20$ $\mathrm{ml} ; \mathrm{b}=0,25 \mathrm{ml} ; \mathrm{c}=0,30 \mathrm{ml}$ et $\mathrm{d}=0,35 \mathrm{ml}$. Le cinquième flacon de chaque série, marqué " Te », a été utilisé comme témoin du développement de la moisissure en l'absence de l'acide acétique. Finalement, tous ces flacons ont été bouchés à l'ouate et incubés à la température ambiante $\left(30^{\circ} \mathrm{C}\right)$ pour suivre le développement du champignon pendant 14 jours.

- Evaluation du poids sec du mycélium formé : Pour en évaluer le poids sec, après filtration des différents milieux liquides sur papier, les mycéliums recueillis ont été bien pressés sur le même support dans des entonnoirs. Les filtrats, recueillis dans les flacons étiquetés, ont été conservés au frais $\left(5^{\circ} \mathrm{C}\right)$ pour une éventuelle recherche d'aflatoxine, tandis que les mycéliums séchés à l'étuve $\left(80^{\circ} \mathrm{C}\right)$ ont été pesés à l'aide d'une balance de précision (APX-200; Denver Instrument).

l'Aspergillus flavus. Et les résultats de ces trois auteurs coïncident avec veux obtenus par 
Mamadou (1978), Nikiema (1993) et Senani (2010).La croissance du mycélium a été premièrement appréciée par une simple observation visuelle de la surface qu'il occupait sur le milieu de culture (Figures 1, 2 et 3).

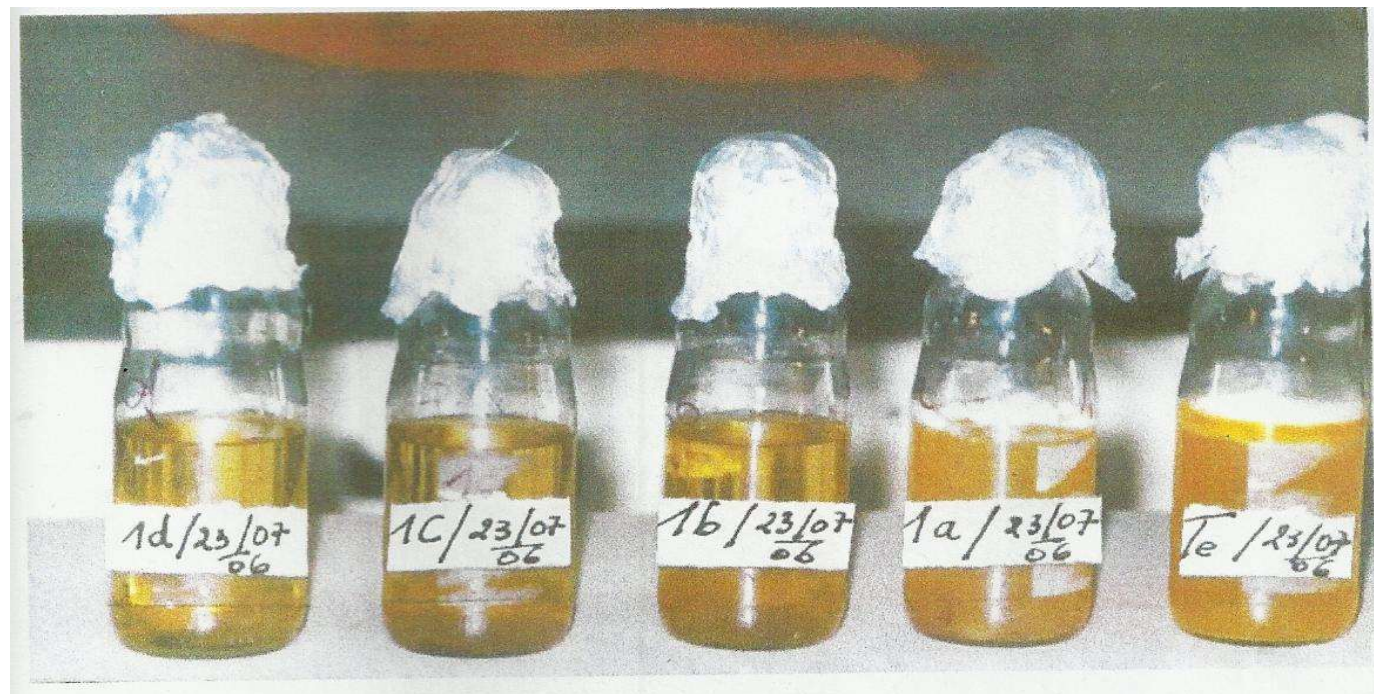

Figure 1 : dans les flacons ld, lc et lb de la première série on constate l'absence du mycélium tandis que dans les flacons la et Te il y a une présence du mycélium. Les milieux de culture inoculés de ces deux derniers flacons deviennent troubles à cause d'un mycélium immergé.

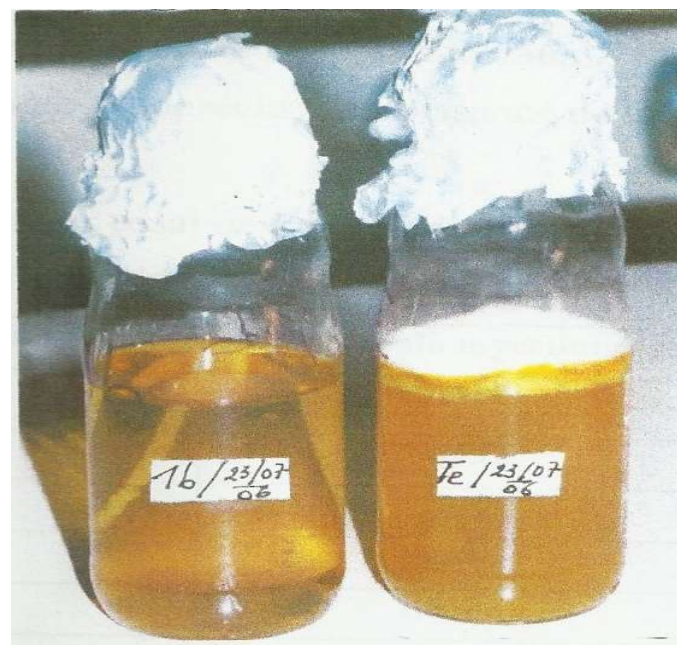

Figure 2 : dans le flacon Te on constate la présence du mycélium, et le milieu de culture est également trouble. Dans le flacon $\mathbf{l b}$ il y a absence du mycélium. 


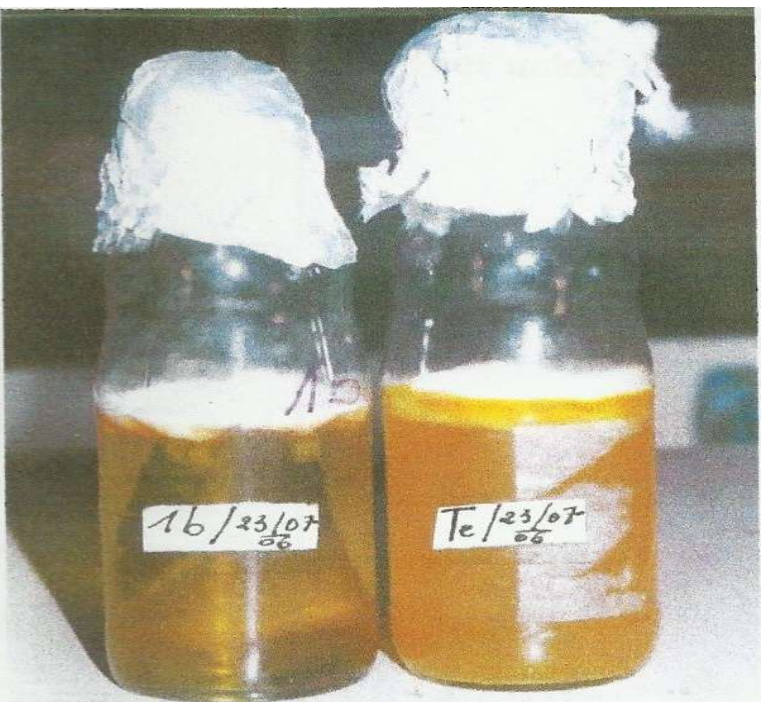

Figure 3 : Pour la deuxième série, on note dans le flacon Te la présence du mycélium, et le milieu de culture est très trouble. Dans le flacon $\mathbf{l b}$ il y a avait plutôt une activation de la croissance du mycélium ; le mycélium y est plus important que dans le témoin.

Après l'observation visuelle, les résultats de l'évaluation du poids sec du mycélium sont représentant donc la seconde manière présentés aux tableaux 5 et 6 ci-après, d'apprécier la croissance de l'Aspergillus flavus.

Tableau 5 : Effet de l'acide acétique sur le développement de la souche d'Aspergillus flavus. (Premier essai)

\begin{tabular}{|c|c|c|}
\hline Traitements & Poids sec du mycélium (g) & $\mathbf{\%}$ \\
\hline $\mathrm{Te}$ & 2,1480 & 100 \\
\hline $\mathrm{T}_{1}$ & 2,0692 & 96,3 \\
\hline $\mathrm{T}_{2}$ & 1,7045 & 79,3 \\
\hline $\mathrm{T}_{3}$ & 1,6865 & 78,5 \\
\hline $\mathrm{T}_{4}$ & 1,6588 & 77,2 \\
\hline
\end{tabular}

Le taux d'inhibition en pourcentage a été calculé en considérant comme équivalant à cent pour cent le développement de la souche d'Aspergillus flavus dans le milieu Czapek témoin. Pour les doses expérimentées, l'inhibition de la croissance a été proportionnelle aux volumes d'acide dans le milieu Czapek et a ainsi varié entre 77,2 et 96,3\%.Dans le second essai avec d'autres volumes d'acide (voir matériels et méthodes), les résultats obtenus sont repris dans tableau 6 ci-après.

Tableau 6 : Effet de l'acide acétique sur le développement de la souche d'Aspergillus flavus.(deuxième essai)

\begin{tabular}{|c|c|c|c|c|c|}
\hline \multirow{2}{*}{ Traitements } & \multicolumn{3}{|c|}{ Poids du mycétique (g) } & Moyenne & \% \\
\hline \multirow{2}{*}{$\mathrm{Te}$} & 1 & 2 & 3 & \multirow{2}{*}{1,167} & \multirow{2}{*}{100} \\
\cline { 2 - 4 } & 1,098 & 1,127 & 1,277 & & \\
\hline $\mathrm{T}_{1}$ & 1,185 & 1,493 & 2,044 & 1,574 & 134,8 \\
\hline $\mathrm{T}_{2}$ & 0,433 & 0,609 & 0,672 & 0,571 & 48,9 \\
\hline $\mathrm{T}_{3}$ & 0,911 & 1,197 & 2,110 & 1,406 & 120,4 \\
\hline
\end{tabular}




\begin{tabular}{c|c|c}
\hline $\mathrm{T}_{4}$ & 0,713 & 0,896 \\
\hline Dans de second essai pour certaines doses
\end{tabular}
expérimentées $\left(T_{2}\right.$ et $\left.T_{4}\right)$, le taux d'inhibition lié à la présence de l'acide acétique dans le milieu Czapek a varié entre 48,9 et 89,2\%. A ces doses inhibitrices la croissance mycélienne d'Aspergillus flavus se traduit par une réduction (Figure1) ou même par l'absence (Figures 1 et 2) du mycélium dans les flacons contenant le même milieu associé à l'acide acétique. Au regard des résultats des tableaux 5 et 6 , il y a lieu de constater que l'acide acétique a un effet antifongique se traduisant par une réduction de la croissance. Cependant, pour les traitements $\mathrm{T}_{1}$ et $\mathrm{T}_{3}$ du second essai (tableau 6 ), il a été plutôt constaté une activation de la croissance mycélienne. A ces deux doses, l'acide acétique a fortement stimulé le développement du champignon. Il peut s'agir d'un artefact

\section{CONCLUSION}

La croissance d'une souche d'Aspergillus flavus en milieu Czapek a été étudiée en présence d'acide acétique. Dans un premier essai, ce champignon a été inhibé proportionnellement aux doses d'acide expérimentées. Dans un second essai, il a été encore observé une inhibition, mais aussi une activation de la croissance mycélienne pour des traitements bien distincts. Cette activation serait certainement due à des facteurs difficiles à déterminer avec précision. Néanmoins il ressort \begin{tabular}{|c|c|c|}
\hline 1,519 & 1,042 & 89,2 \\
\hline expérimental. & Le développement des
\end{tabular} moisissures est aussi fonction des proportions de dioxygène, de diazote et de dioxyde de carbone présents dans les flacons bouchés à l'ouate, ce dernier pouvant permettre certains échanges gazeux. En effet, la croissance mycélienne est multipliée par deux lorsque la teneur en oxygène est augmentée à plus de 0,56 $\%$. De plus, les interactions entre les gaz et l'humidité influencent le développement des moisissures Il s'avère également que des ensemencements consécutifs de la même souche à partir de la même culture mère, et cela dans des conditions expérimentales identiques, pourraient entretenir la prolifération du champignon suite à une éventuelle adaptation qui peut être réduite lorsqu'on procède à un mélange entre ces différents ensemencements.

de ces travaux que l'acide acétique freine le développement de l'Aspergillus flavus et pourrait donc être exploité dans le cadre de la lutte contre ce champignon dans certaines denrées. La dose limite efficace, dans nos essais, peut être estimée à 98,9\% ppm. Les conditions du milieu, surtout lorsqu'on travaille en milieu liquide comme ce fut cecas, sont difficiles à circonscrire étant donné l'interaction de plusieurs paramètres non contrôlés.

\section{REFERENCES BIBLIOGRAPHIQUES}

Allcroft, R., Rogeres, H., Lewis, G., Nabney, J. et Best, P. 1966 Metabolism of aflatoxin in sheep; excretion of the "mill toxin". Nature, 209, 154-155 pp.

Bastiaanse H, et Van Hove F, 2006 Caractérisation des espèces de champignons et de mycotoxines contaminant des ensilages en Belgique, $6 \mathrm{p}$.

Boucher, S. - Nouaille, L. 2002 Maladies des lapins, $2^{\text {ème }}$ édition revu et mise à jour. Paris, France Agricole 271 p.

Clavel, D. 2005 Atelier final du projet "New Tools for groundnut aflatoxin control in Sahel Africa" Centre de coopération internationale en recherche agronomique pour le développement. 1 p.

Dalezios, J., Wogan, G. et Weinreb, S. AflatoxinP1: a new aflatoxin metabolite in monkeys. Science, 171, 584-585 pp.

DeWale, D. Swanelder, C.J. 2001, "Arachide", dans Raemaekers, R.H. (Coord.) p.767785 
Diafuka N. J.B., 2006-2007 Effets inhibiteurs des extraits des rhizomes de Zingiber officinale te des extraits de Capsicumfrutescens dans le développement de l'Aspergillus flavus et la production des aflatoxines, Travail de Fin d'Études ISAV, inédit, $46 \mathrm{p}$.

Dieme E, Fall R, Sarr I, Sarr F, Traore D, et Seydi M., 2016 Contamination des cereals par l'aflatoxine en Afrique: revue des méthodes de lutte existantes. In Int J. Biol. Chem. Sci10 (5) : 2285-2299 pp.

FAO, 1989 www.fao.org/3/x5036e/x5036 e0q.htm, www.fao.org/docrep/x5036e $\angle \mathrm{x} 5036 \mathrm{e} 08 . \mathrm{htm}$

GolamaSwana, 2005 Systématique des végétaux inférieurs. Notes de cours, ISP/GOMBE, inédit

Griscellic, Mouyr\&Vilmer, 1984 infection Aspergillaire et granulomatose septique chronique, Médecine te maladies infectieuses, $566 \mathrm{p}$.

Ivami Emeka C. 2006-2007 Inhibition de l'Aspergillus par l'acide acétique, Mémoire d'Ingénieur, faculté des Sciences Agronomiques de l'Université de Kinshasa, Travail de Fin de Cycle, inédit, $33 \mathrm{p}$.

Khasa M., 2006 Inhibition de l'Aspergillus flavus par l'acide proprionique, Faculté des Sciences Agronomiques de l'Université de Kinshasa Travail de Fin de Cycle, inédit, $33 \mathrm{p}$.

Luyila M. M 2005-2006 Action inhibitrice de l'acide acétique sur le développement de l'Aspergillus flavusresponsable de la toxicité de quelques aliments consommés en RD Congo. Mémoire de Licence, ISP/Gombe, inédit, 43 p.

Makuba L., 2006-2007 Effets inhibiteurs des extraits de Moringa oléifera et de Citrus grandis sur le développement de l'Aspergillus flavus et sur la production des aflatoxines, T.F.E., ISAV/KIMLWENZA, inédit, 35 p.
Mamadou D, 1978 Étude des problèmes posés par les aflatoxines dans les aliments du bétail et de l'homme. Thèse de doctorat, Écoles Inter-états des Sciences et Médecine Vétérinaires, Dakar, 93 pages

Manima P., 2004-2005 Contribution à la lutte microbiologique contre les Aflatoxines - Antagonisme entre Aspergillus flavus et proprioni bacteriumacne, Mémoire d'Ingénieur, Faculté des Sciences Agronomiques de l'Université de Kinshasa, inédit, $29 \mathrm{p}$.

Masimango, N.T., 1978 Contribution à l'étude de la production de l'aflatoxine B1 par Aspergillus flavus Link-Lutte contre la présence de cette toxine dans certaines denrées, Thèse de doctorat, Faculté des Sciences Agronomiques de l'État, Gembloux, 222 p.

Masimango, N.T., 1987 Complément de Biologie, Syllabus, UNIKIN, inédit, 46 p.

Mazoyer, M., 2002 Larousse Agricole. Le paysan au XXI ${ }^{\text {ème }}$ Siècle, Montréal Larousse, $767 \mathrm{p}$.

Moll, M. \& Moll, N., 2002, Précis des risques alimentaires. Technique et Documentation, Paris, 383 p.

Muhuruka B., 2005, Effet de l'acide acétique sur le développement de l'Aspergillus flavus et la production de l'Aflatoxine, TFC Faculté des Sciences Agronomiques, UNIKIN, inédit, 27 p.

Nikiema A, 1993 Étude des aflatoxines au Burkina Faso: Détermination quantitative et qualitative des aflatoxines de l'arachide par des tests biochimiques et immunologiques. Thèse de doctorat de spécialité en Sciences Biologiques Appliquées, Faculté des Sciences et Techniques, Université de Ouagadougou, 118 p.

Pitt, J.l. and Hocking, A.D (1985a) Fungi and Food Spoilage. Sydney, N.S.W. : Academic Press. 
Pululu M.N., 2005, Questions approfondies de Botanique. Notes de cours ISP/GHOMBE, inédit, $57 \mathrm{p}$.

Reddy DVR, Thirumala-Devi K, Reddy SV, Waliyar F, Mayo M A, RamaDevi K, Ortiz R and Lenne JM, 2002 Estimation des niveaux d'aflatoxines dans certaines denrées alimentaires et dans l'alimentation animale en Inde. Éds HANAK E, BOUTRIF E et FABRE P. Gestion de la sécurité des aliments dans les pays en développement. Actes de l'atelier international, CIRAD-FAO, $4 \mathrm{p}$.

Sargeant, K., Sheridan, A.O., Kelly, J. \&Karnaghan, R., 1961, Toxicity associated with certain samples groundnuts. Nature 192: 1096-1097 pp.

Senani N, 2010 Étude du pouvoir antifongique (inhibition de croissance et de production d'aflatoxines) des composés et extraits polyphenoliques issus des olives et sous-produits de l'olivier (feuilles et margines) variété chamtalsur deux souches, Aspergillus flavuset Aspergillusparasiticus. Mémoire de magister en Sciences biologiques, Faculté des Sciences Biologiques et des sciences Agronomiques, Université Mouloud Mammeri de Tizi-Ouzou, 142 $\mathrm{p}$

Simba Amina, M., 2006 Effet de l'acide proprionique sur la croissance mycélienne et la production des aflatoxines d'Aspergillus flavus, Mémoire de Licence ISP/GOMBE, inédit, 25 p.

Stevenson, D. 1961, Toxicity associated with certain bactches of groundnuts. Bultveter. J. 118:531-532 pp.

Sung-Hye Cho, Chang-Hee Lee, Mi-Ran Jang, Young-Wook Son, Sang-Mok Lee, InSun Choi, So-Hee Kim et Dai-Byung Kim, 2008 Aflatoxins contamination in spices and processed spice products commercialized in Korea. Analytical Methods. Food Chemistry, 107, 1283-1288 pp.
Umba, J. M. 2004 Production d'agents antifongiques par culture de cellules immobilisées sur supports solides et étude de leur application dans le contrôle des moisissures (productrices d'aflatoxines) contaminant certains produits alimentaires. Projet de thèse de doctorat en Sciences agronomiques, Faculté des Sciences Agronomiques, Département de Chimie et Industries Agricoles, Université de Kinshasa, inédit, $10 \mathrm{p}$.

Vesely D, Vesela D, Jelinek R, 1983 Comparative assessment of the aflatoxin $B_{1}, B_{2}, G_{1}, G_{2}$ et M1 embryo toxicity in the chick embryo, Toxicology Letters, 15, 297-302 pp. 